\title{
CNNs Fusion for Building Detection in Aerial Images for the Building Detection Challenge
}

\author{
Remi Delassus \\ Qucit, 213 cours Victor Hugo, 33130 Begles, France \\ Univ. Bordeaux, Bordeaux INP, CNRS, LaBRI, UMR5800, F-33400 Talence, France \\ remi.delassusequcit.com \\ Romain Giot \\ Univ. Bordeaux, Bordeaux INP, CNRS, LaBRI, UMR5800, F-33400 Talence, France \\ romain.gioteu-bordeaux.fr
}

\begin{abstract}
This paper presents our contribution to the DeepGlobe Building Detection Challenge. We enhanced the SpaceNet Challenge winning solution by proposing a new fusion strategy based on a deep combiner using segmentation both results of different $C N N$ and input data to segment. Segmentation results for all cities have been significantly improved (between 1\% improvement over the baseline for the smallest one to more than $7 \%$ for the largest one). The separation of adjacent buildings should be the next enhancement made to the solution.
\end{abstract}

\section{Introduction}

The DeepGlobe Building Detection Challenge [5] follows the second round of the SpaceNet Challenge 1 . It poses the challenge of automatically detecting buildings from satellite images

Top competitors of the SpaceNet challenge have submitted their code which is now publicly available ${ }^{2}$ We thus studied the winning solution that consists in an ensemble of three U-Net [20] based models whose segmentation results are averaged, further called the "baseline".

A model built by merging the prediction of multiple sub models, like this one, is called an ensemble model; it generally performs better than the best of the merged models [12]. The most common merging strategy for image segmentation is an unweighted average of the predictions.

We propose to replace the original unweighted average

${ }^{1}$ https: / / community $\cdot$ topcoder. com/tc?module= MatchDetails\&rd=16892

2 https://github.com/SpaceNetChallenge/ BuildingDetectors_Round2 by another ensemble model that we a call a deep combiner. Its architecture is equivalent to the combined models (i.e, $\mathrm{U}$ Net based) and works with a MUL input as well as predicted segmentations. This combiner improves the baseline from $1 \%$ in Vegas to $7.4 \%$ in Khartoum.

This paper is organized as follows. Section 2 presents previous works on building detection and fusion techniques. We see here the existing building extraction methods, existing work on models' output fusion for segmentation, and SpaceNet's winning solution presentation. Section 3 presents our deep combiner. Section 4 shows the results; the combiner enhance the segmentation and the detection of buildings. Section 5 concludes this work.

\section{Previous work}

We based our model on the winning solution of the SpaceNet challenge. it used an unweghted average based ensemble of 3 U-Net models to segment buildings from the 8 MUL channels of the input Image as well as OpenStreetMap data. The contours of the building are then extractedd from the segmentation by grouping together into one building all connected pixels.

\subsection{Limitations of the baseline}

Several limits of the baseline appear. Firstly, the models are trained to predict a mask, which is not optimal. When two buildings are too close to each other, the contours of the buildings cannot be extracted from the corresponding mask (Figure 3) and the two buildings are considered as one.

Secondly, the post-processing is close to non existent. For example, one could use an instance segmentation model [2] or a flooding algorithm [19] to split adjacent buildings. Those adjacent buildings will be fused into one single building if a group of at least one pixel adjacent 
to both of them is classified as a group of building pixels. Another example of what can be done during the post processing phase is the smoothing of building edges. Because most of the time, buildings have regular shapes, we can use this knowledge to predict shapes closer to reality. CRF [13] techniques have been used to smooth edges and enhance the resolution of segmentations. It could help sharpening polygons and separating them.

Thirdly, more recent architectures such as DeepLab [3] or PSPnet [24] could be used to try to enhance the segmentation score. They outperform the U-Net architecture in ImageNet [21] segmentation tasks. However, their strong performance comes from the ability to detect tiny objects as well as big ones, which may not be so useful in the building detection problem.

Finally, a simple unweighted average is often a good default choice, as it will reduce the variance of the result, but might not be the best fusion solution as it gives the same importance to all models regardless of their performance, and is subject to errors when one of the merged models is overconfident, or when a large proportion of the merged models is erroneous. This critical proportion is easily reached when only three models are merged.

This study focuses on this final limitation. We thus review the state of ensemble modeling applied to problems of image segmentation.

\subsection{Ensemble modeling for image segmentation}

Ju et al [9] show that unweighted average (used by Marmanis et al [18] on multiple instances of the same model architecture and by Kamnitsas et al [10] on complementary architectures) is a fusion method that performs as good as, if not better than, other known methods. It is favorable for reducing variance among predictions, but suffers from overconfident models. Majority voting (used by Dolz et al [6]) generally has a lower performance pixel wise, but can produce an uncertainty map, which can be used to focus the model training on uncertain areas [22], or to prevent uncertain pixel classifications [11] which could help differentiate buildings close to each other and lead to worthier performances in building detection.

Weighted average with learned weights is called by $\mathrm{Ju}$ et al [9] a super learner. It is a model that takes segmentation as an input and outputs a more advantageous one. The simplest one can be seen as $1 * 1 * m$ convolution where $m$ is the number of models fusionned. In their study on the CIFAR10 [14] dataset, they show that it usually performs better than unweighted averages but does not investigate more complex super learners, such as the one studied here; super learners taking neighborhood into account, or deep super learners. They do not talk about non learned weights, such as weighs accordingly proportional to the models' performances [15], but we can reasonably assume that those weights would be reached by learn methods if they happened to be optimal, simply by setting them as initial weights.

The success of residual networks [7] shows that learning residual functions with reference to the layer inputs, instead of learning unreferenced functions can lead to better performance. Scaled up to the model instead of the layer, we study a model that will use the input image as well as the fused models' outputs to learn a better segmentation. This additional input puts our model outside of the super learner category. We will label as combiners those models dealing with combining the output of other models, with or without additional outputs.

\section{Proposed combiner}

We propose a deep learning approach to the fusion of segmentation: a U-net based deep combiner that combines the segmentations output of the combined models as well as the MUL channels of the input image.

\subsection{Architecture}

The Deep combiner approach aims to enhance the segmentation by letting the deep neural network compute complex features such as edge detection, distance to building pixels etc. Moreover, this combiner uses not only the three predicted segmentations, but also the original image as input. This image can be used by the combiner model to determine the residuals of input models and fix their mistakes. We use the MUL channels of the original image as input.

We use the same U-Net architecture as input networks, with 29 hidden layers and 7838273 parameters. This architecture is usually used to segment images. Combining segmentations into one can be seen as performing a new segmentation on an image in which some channels correspond to some proposed segmentations. It thus makes sense to use a segmentation architecture to perform the combination.

This combiner was not manually initialized to perform the unweighted average of the previous segmentation as it would jeopardize the learning process. Weigths are initialized with a uniform distribution between -0.05 and +0.05 . We name this combiner the $U$-net approach.

\subsection{Training}

Training data is augmented, as we don't have enough images for some cities to train a deep learning model. For example, we train the model for Khartoum on 708 images and validate it on 304. The additional data comes from the rotation of the original image of 90, 180 and 270 degrees, as well as a symmetry along both axis.

We train our combiner during 20 epochs with a batch size of 1 . We selecte the best epoch based on the validation score. Optimal validation score is usually reached between 
Table 1. F-score, of the baseline and U-Net combiners over the validation set (including U-Net gain over baseline) compared to U-Net combiner final score for this challenge.

\begin{tabular}{|l|l|l|c|}
\hline City & Baseline & U-Net & U-Net final score \\
\hline Vegas & 0.8559 & 0.8639 & 0.8057 \\
& - & $+0.93 \%$ & \\
\hline Paris & 0.6805 & 0.7080 & 0.6787 \\
& - & $+4.04 \%$ & \\
\hline Shanghai & 0.5627 & 0.5794 & 0.5661 \\
& - & $+2.97 \%$ & \\
\hline Khartoum & 0.5855 & 0.6290 & 0.6387 \\
& - & $+7.43 \%$ & \\
\hline
\end{tabular}

epoch 5 and epoch 10 . We evaluate the segmentation using the Jaccard coefficient [8] for our loss function. It is the closest metric to the IoU score (used to judge if two polygons match) when it comes to image comparison. Given a ground truth labeling $y^{*}$ and a predicted segmentation to be evaluated $\hat{y}$, the Jaccard coefficient is defined as:

$$
J\left(y^{*}, \hat{y}\right)=\frac{y^{*} \cap \hat{y}}{y^{*} \cup \hat{y}}
$$

which for images translates into:

$$
J\left(y^{*}, \hat{y}\right)=\frac{\sum\left(y^{*} * \hat{y}\right)}{\sum\left(y^{*}+\hat{y}\right)-\sum\left(y^{*} * \hat{y}\right)}
$$

With the assumption that $J(0,0)=1$. As the Jaccard coefficient produces a value between 0 and 1 , it is straightforward to transform it into a loss function:

$$
J_{l}\left(y^{*}, \hat{y}\right)=1-J\left(y^{*}, \hat{y}\right)
$$

Experiments have been run on a Linux computer with the following characteristics: Intel(R) Core(TM) i7-6950X CPU@ @ 3.00GHz, three Titan X Pascal GPUs (used to train multiple model in parallel, with one model per GPU) and 120Gbits of RAM, using Keras [4] 1.2.2 with Tensorflow [1] 1.4.0.

\section{Results}

\subsection{F-score improvement}

Table 1 reports the maximum value of F-score obtained on each city dataset with the baseline and the combiner. The gain is defined as Gain = (New_Score Baseline)/Baseline. There has only been a slight improvement on Vegas because the baseline is already performing extremely well. However a large improvement on Khartoum shows that our solution can address more cases than a simple unweighted average.

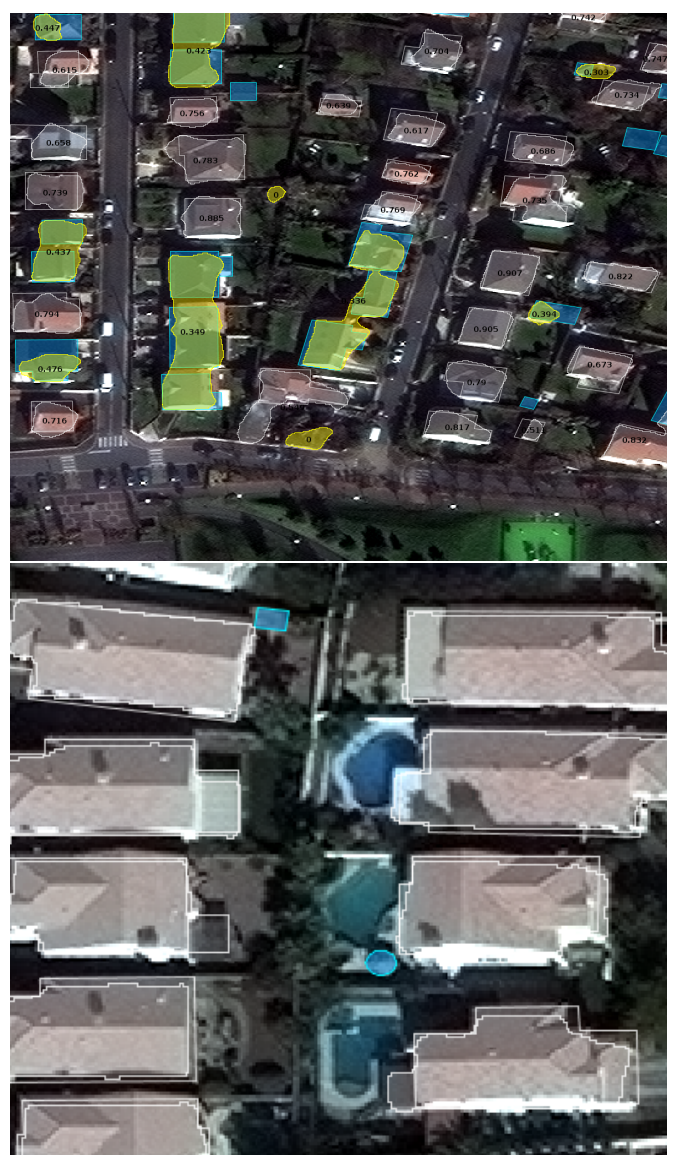

Figure 1. Example of errors. In the first image adjacent buildings are fused because they were too close from each other. In the second image only the big buildings have been detected while the little roofs have not.

\subsection{Visualization of Detected Buildings}

Images in Figures 1 and 2 have been produced with the SpaceNet Challenge Building Detector Visualizer 3 Ground truth and predicted polygons are overlaid over the RGB images. Matching ground truth and predicted polygons are white. Yellow polygons are false positives (predicted polygon with no matching ground truth). Blue polygons are false negatives (ground truth polygon with no matching prediction).

A vast majority of buildings are properly segmented. Only buidings with a surface area significantly smaller than surrounding buildings are ignored by the combiner. The major difficulty is to separate buildings too close from each other.

\subsection{Discussion}

We have been able to highlight two main sources of errors. The first one is the adjacent buildings problem (Fig-

$\sqrt[3]{\text { https://github.com/SpaceNetChallenge/ }}$ BuildingDetectorVisualizer 


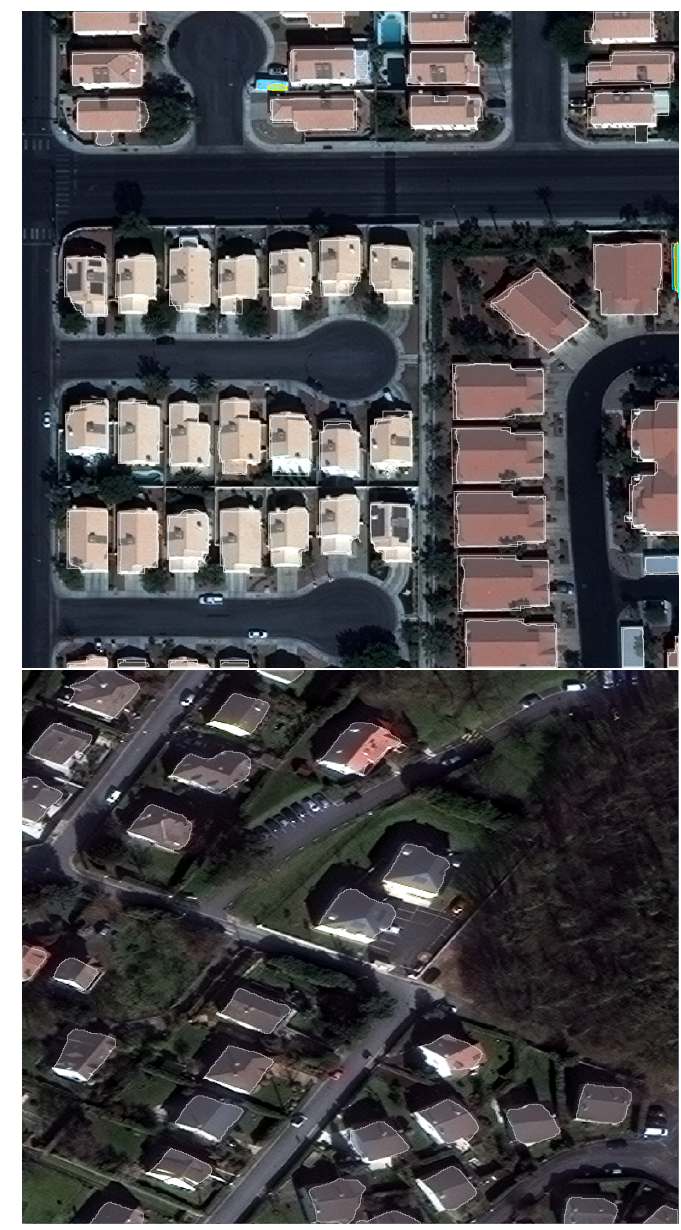

Figure 2. Example of high building density images. We can see that a large majority of the buildings are correctly detected. The few errors come from the smaller buildings, or from small parts of buildings cut at the edges of the image, as well as adjacent buildings being merged into one.

ure 3). When we reduce the model uncertainty around buildings, adjacent buildings are considered as one unique building instead of two different ones. The second one comes from numerous errors in labeled data. Those errors can prevent the models to learn useful features or can punish them when they do. Missing a lot of small buildings will have a huge impact on the F-score, but smaller buildings are not consistenly labeled, especially in Shanghai.

To solve the adjacent buildings problem, Marmanis et al [17] introduce the border class, such that the model classifies each pixel in three classes: building, building border, and background. It is then easier to split adjacent buildings. Yuan et al [23] use a distance field as label. Each pixel value is its distance to the closest border (negative when inside a building). It is then possible to configure the postprocessing and set a predicted distance ( 0 in the mask) as the border. But it requires a large receptive field for this dataset.

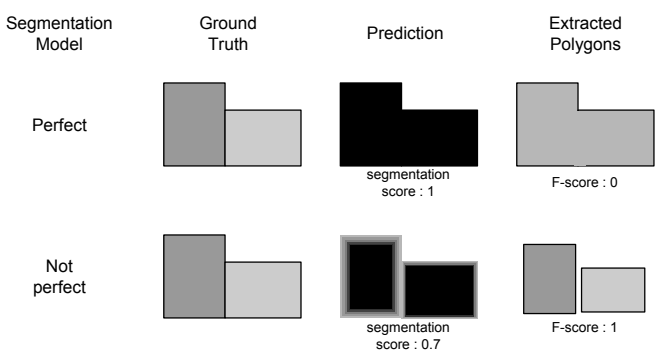

Figure 3. Adjacent buildings problem. When two buildings are too close to each other, a perfect model will produce only one building if the chosen label is a mask.

We used the same architecture for the combiner as the one used for the combined models. More architectures should be tested to determine if regular convnets are the best combiners. Maggiori et al [16] used a recurrent neural network to improve their segmentation, which is another solution. Bai et al [2] used a new architecture of convnets to perform an instance segmentation. Their model takes the input image and the result of a segmentation as an input, just like our U-net.

The deep combiner was only tested with the combined learned output segmentation and the MUL input. The OSM layers and multi band inputs were not used. Of the three combined learners, the best ones don't use RGB, but multiband image and OSM layers. A deep combiner using those inputs is likely to have better performances too and will thus be implemented once the adjacent buildings' problem will be solved.

\section{Conclusion}

This paper addresses the problem of segmenting buildings from aerial images. It proposes a fusion solution for an ensemble of U-net models used to segment aerial images and extract buildings' contours from the segmentation. We used SpaceNet's challenge winning solution as a baseline to evaluate the benefits of combiners over unweighted averages for model fusion. This baseline corresponds to an ensemble of three models with the same architecture but different inputs. For two of the three studied images set (Vegas and Khartoum), a better segmentation led to a better Fscore, and combiners were thus performing superiorly than the existing unweighted averages. For the Paris dataset, the enhancement of the segmentation led to a worsened F-score, showing that the separation of adjacent buildings should be the next part of the solution to enhance.

Future work will focus on adjacent buildings' separation, thanks to an advantageous label such as the addition of a building borders class, or through post processing of the segmentation, as proposed by Bai et al [2]. 


\section{References}

[1] M. Abadi, A. Agarwal, P. Barham, E. Brevdo, Z. Chen, C. Citro, G. S. Corrado, A. Davis, J. Dean, M. Devin, et al. Tensorflow: Large-scale machine learning on heterogeneous distributed systems. arXiv preprint arXiv:1603.04467, 2016.

[2] M. Bai and R. Urtasun. Deep watershed transform for instance segmentation. In CVPR, 2017, pages 2858-2866. IEEE, 2017.

[3] L.-C. Chen, G. Papandreou, I. Kokkinos, K. Murphy, and A. L. Yuille. Deeplab: Semantic image segmentation with deep convolutional nets, atrous convolution, and fully connected crfs. arXiv preprint arXiv:1606.00915, 2016.

[4] F. Chollet et al. Keras. https://github.com/ fchollet/keras 2015.

[5] I. Demir, K. Koperski, D. Lindenbaum, G. Pang, J. Huang, S. Basu, F. Hughes, D. Tuia, and R. Raskar. Deepglobe 2018: A challenge to parse the earth through satellite images. arXiv preprint arXiv:1805.06561, 2018.

[6] J. Dolz, C. Desrosiers, L. Wang, J. Yuan, D. Shen, and I. B. Ayed. Deep cnn ensembles and suggestive annotations for infant brain mri segmentation. arXiv preprint arXiv:1712.05319, 2017.

[7] K. He, X. Zhang, S. Ren, and J. Sun. Deep residual learning for image recognition. In Proceedings of the IEEE conference on computer vision and pattern recognition, pages 770-778, 2016.

[8] P. Jaccard. Distribution de la flore alpine dans le bassin des dranses et dans quelques régions voisines. Bull. Soc. Vaud. Sci. Nat., 37:241-272, 1901.

[9] C. Ju, A. Bibaut, and M. J. van der Laan. The relative performance of ensemble methods with deep convolutional neural networks for image classification. arXiv preprint arXiv:1704.01664, 2017.

[10] K. Kamnitsas, W. Bai, E. Ferrante, S. McDonagh, M. Sinclair, N. Pawlowski, M. Rajchl, M. Lee, B. Kainz, D. Rueckert, et al. Ensembles of multiple models and architectures for robust brain tumour segmentation. arXiv preprint arXiv:1711.01468, 2017.

[11] M. Kampffmeyer, A.-B. Salberg, and R. Jenssen. Semantic segmentation of small objects and modeling of uncertainty in urban remote sensing images using deep convolutional neural networks. In Proceedings CVPR Workshops, pages 1-9, 2016.

[12] J. Kittler, M. Hatef, R. P. W. Duin, and J. Matas. On combining classifiers. IEEE PAMI, 20(3):226-239, Mar. 1998.
[13] P. Krähenbühl and V. Koltun. Efficient inference in fully connected crfs with gaussian edge potentials. In Advances in neural information processing systems, pages 109-117, 2011.

[14] A. Krizhevsky and G. Hinton. Learning multiple layers of features from tiny images. 2009.

[15] A. Lahiri, A. G. Roy, D. Sheet, and P. K. Biswas. Deep neural ensemble for retinal vessel segmentation in fundus images towards achieving label-free angiography. In Engineering in Medicine and Biology Society (EMBC), 2016 IEEE 38th Annual International Conference of the, pages 1340-1343. IEEE, 2016.

[16] E. Maggiori, G. Charpiat, Y. Tarabalka, and P. Alliez. Recurrent neural networks to correct satellite image classification maps. IEEE TGRS, 2017.

[17] D. Marmanis, K. Schindler, J. D. Wegner, S. Galliani, M. Datcu, and U. Stilla. Classification with an edge: improving semantic image segmentation with boundary detection. arXiv preprint arXiv:1612.01337, 2016.

[18] D. Marmanis, J. D. Wegner, S. Galliani, K. Schindler, M. Datcu, and U. Stilla. Semantic segmentation of aerial images with an ensemble of cnss. ISPRS Annals of the Photogrammetry, 2016, 3:473-480, 2016.

[19] F. Meyer. Color image segmentation. In Image Processing and its Applications, 1992., International Conference on, pages 303-306. IET, 1992.

[20] O. Ronneberger, P. Fischer, and T. Brox. U-net: Convolutional networks for biomedical image segmentation. In MICCAI, pages 234-241. Springer, 2015.

[21] O. Russakovsky, J. Deng, H. Su, J. Krause, S. Satheesh, S. Ma, Z. Huang, A. Karpathy, A. Khosla, M. Bernstein, A. C. Berg, and L. Fei-Fei. ImageNet Large Scale Visual Recognition Challenge. International Journal of Computer Vision (IJCV), 115(3):211-252, 2015.

[22] H. Wang, Y. Wang, Q. Zhang, S. Xiang, and C. Pan. Gated convolutional neural network for semantic segmentation in high-resolution images. Remote Sensing, 9(5):446, 2017.

[23] J. Yuan. Learning building extraction in aerial scenes with convolutional networks. IEEE transactions on pattern analysis and machine intelligence, 2017.

[24] H. Zhao, J. Shi, X. Qi, X. Wang, and J. Jia. Pyramid scene parsing network. arXiv preprint arXiv:1612.01105, 2016. 